Journal of Mathematics and Informatics

Vol. 11, 2017, 83-93

ISSN: 2349-0632 (P), 2349-0640 (online)

Published 11 December 2017

www.researchmathsci.org

DOI: http://dx.doi.org/10.22457/jmi.v11a11

Journal of

Mathematics and

Informatics

\title{
Degree Based Topological Indices of Zig Zag Chain
}

\author{
P.Gayathri ${ }^{1}$ and U.Priyanka ${ }^{2}$
}

PG and Research Department of Mathematics

A.V.C.College (Autonomous), Mannampandal, Mayiladuthurai,Tamilnadu, India

Email: ${ }^{1}$ pgayathrisundar@gmail.com; ${ }^{2}$ upavc95@gmail.com

Received 6 November 2017; accepted 8 December 2017

Abstract. In this paper, we obtained the degree based topological indices like Randic, Geometric - Arithmetic, Sum Connectivity, Harmonic, First Zagreb, Second Zagreb, Second Modified Zagreb, Inverse Sum, Albertson, Atom - bond Connectivity, Symmetric - Division index and Augmented Zagreb indices for Zig-Zag chain of 8cycles molecular graph. The degree based topological indices for the Zig- Zag chain of 8cycles are computed as expressions in ' $a$ ' and ' $n$ ', where ' $a$ ' represents the number of hexagons and 'n' represents the number of segments.

Keywords: Topological index, molecular graph, M-polynomial, zig-zag chain of 8cycles.

AMS Mathematics Subject Classification (2010): 05C05, $05 \mathrm{C} 12$

\section{Introduction}

In medicine mathematical model, the structure of drug is represented as an undirected graph, where each vertex indicates an atom and each edge represents a chemical bond between these atoms.

With rapid development of medicine manufacture, a large number of new drugs have been developed each year. Hence, it demands a tremendous amount of work to determine the pharmacological, chemical and biological characteristics of these new drugs, and such workloads become more and more fussy and clustered.

It requires enough reagents equipment and assistants to test the performances and the side effects of existing new drugs. However, in lower income countries and areas (such as certain cities and countries in South America, Africa and Southeast Asia), there is no sufficient money to defray reagents and equipment which can be employed to measure the biochemical properties.

Fortunately, many previous studies[1-4] have pointed that chemical and pharma codynamics characteristics of drugs and their molecular structures are closely linked. If we calculate indicators of these drug molecular structures[5-9] in view of defining the topological indices, the medical and pharmaceutical scholars could find it useful to well know their medicinal properties, which can make up the defects of medicine and chemical experiments. From this standpoint, the methods on topological index computation are very suitable and serviceable for developing countries in which they can 


\section{P.Gayathri ${ }^{l}$ and U.Priyanka ${ }^{2}$}

yield the available biological and medical information of new drugs without chemical experiment hardware.

Although there have been several contributions on distance-based indices and degreebased molecular structures,[10-13] the researches of topological index for certain special drug structures are still largely limited. Because of these, tremendous academic and industrial interest has been attracted to research the topological index of drug molecular structure from a mathematical point of view.

\section{Computational procedure of M-polynomial}

M-Polynomial of graph $\mathrm{G}$ is defined as if $G=(V, E)$ is a graph and $v \in V$, then $d_{v}(G)$ (or $d_{v}$ for short if $\mathrm{G}$ is clear from the context) denotes the degree of $\mathrm{v}$. Let $\mathrm{G}$ be a graph and let $m_{i j}(G), i, j \geq 1$, be the number of edges $\mathrm{e}=\mathrm{uv}$ of $\mathrm{G}$ such that $\left\{d_{u}(G), d_{v}(G)\right\}=\{i, j\}$ The M-polynomial of $\mathrm{G}$ as $M(G ; x, y)=\sum_{i \leq j} m_{i j}(G) x^{i} y^{j}$.For a graph $G=(V, E)$, a degree-based topological index is a graph invariant of the form $I(G)=\sum_{e=u v \in E} f\left(d_{u}, d_{v}\right)$ where $f=f(x, y)$ is a function appropriately selected for possible chemical applications.

\section{Some special cases}

\subsection{Case 1}

Let ' $n$ ' be the number of rows in the Zig- Zag chain of 8- cycles $Z_{8}(n)$, for $n=1$ $\Rightarrow$ Number of hexagon $=\mathrm{a}=2$

For $Z_{8}(1)$, the number of edges with end degrees $(2,2)$ is 10 , the number of edges with end degrees $(2,3)$ is 4 , the number of edges with end degrees $(3,3)$ is 1 , total number of edges for $\mathrm{n}=1$ is 15 .

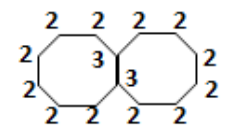

\subsection{Case 2}

Let ' $n$ ' be the number of rows in the Zig- Zag chain of 8- cycles $Z_{8}(n)$, for $n=2$

$\Rightarrow$ Number of hexagon $=\mathrm{a}=4$

For $Z_{8}(2)$, the number of edges with end degrees $(2,2)$ is 16 , the number of edges with end degrees $(2,3)$ is 8 , the number of edges with end degrees $(3,3)$ is 5 , total number of edges for $\mathrm{n}=2$ is 29 .

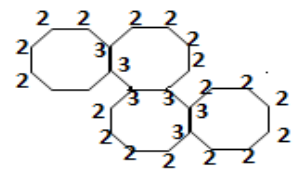

\subsection{Case 3}

Let ' $n$ ' be the number of rows in the Zig- Zag chain of 8- cycles $Z_{8}(n)$, for $n=3$

$\Rightarrow$ Number of hexagon $=\mathrm{a}=6$ 
Degree based topological indices of zig zag chain

For $Z_{8}(3)$, the number of edges with end degrees $(2,2)$ is 22 , the number of edges with end degrees $(2,3)$ is 12 , the number of edges with end degrees $(3,3)$ is 9 , total number of edges for $n=3$ is 43 .

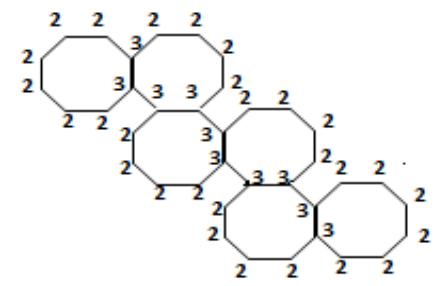

\subsection{Case 4}

Let ' $n$ ' be the number of rows in the Zig- Zag chain of 8- cycles $Z_{8}(n)$, for $n=4$ $\Rightarrow$ Number of hexagon $=\mathrm{a}=8$

For $Z_{8}(4)$, the number of edges with end degrees $(2,2)$ is 28 , the number of edges with end degrees $(2,3)$ is 16 , the number of edges with end degrees $(3,3)$ is 13 , total number of edges for $\mathrm{n}=4$ is 57 .

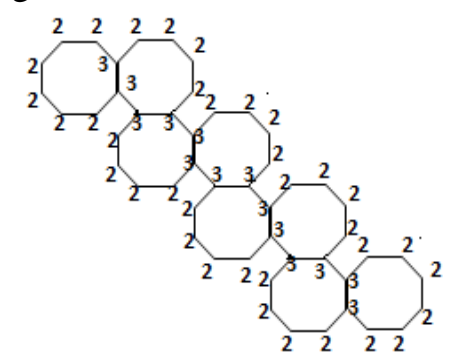

\subsection{Case 5}

Let ' $n$ ' be the number of rows in the Zig- Zag chain of 8- cycles $Z_{8}(n)$, for $n=5$

$\Rightarrow$ Number of hexagon $=\mathrm{a}=10$

For $Z_{8}(n)$, the number of edges with end degrees $(2,2)$ is 34 , the number of edges with end degrees $(2,3)$ is 20 , the number of edges with end degrees $(3,3)$ is 17 , total number of edges for $\mathrm{n}=5$ is 71 .

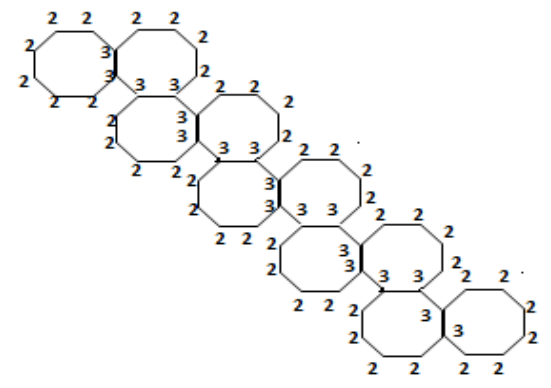

\section{Generalization of zig zag chain of molecular graph}

For $Z_{8}(n)$, for $n \geq 1, a=2 n$ then the number of edges with end degrees $(2,2)$ is $6 n+4$, the number of edges with end degrees $(2,3)$ is $4 \mathrm{n}$, the number of edges with end degrees $(3,3)$ is $4 n-3$, total number of edges for any $n$ is given by $14 n+1$. 


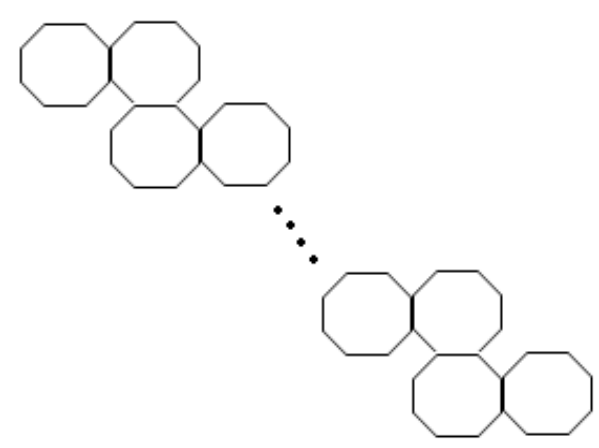

\section{M-Polynomial of ZigZag chain of 8-cycles of graph}

Let

$$
\mathrm{Z}_{8}(\mathrm{n})=(6 n+4) x^{2} y^{2}+(4 n) x^{2} y^{3}+(4 n-3) x^{3} y^{3} \text { for all } n \geq 1
$$

\begin{tabular}{|l|l|l|l|l|l|}
\hline $\begin{array}{l}\text { Topological } \\
\text { Index }\end{array}$ & $\left\{\varphi_{i j}\right\}$ & Notation & $\begin{array}{l}\text { Topological } \\
\text { Index }\end{array}$ & $\left\{\varphi_{i j}\right\}$ & Notation \\
\hline Randic & $\frac{1}{\sqrt{i j}}$ & $\chi(G)$ & $\begin{array}{l}\text { Second } \\
\text { modified } \\
\text { Zagreb }\end{array}$ & $\frac{1}{i j}$ & $M_{3}(G)$ \\
\hline $\begin{array}{l}\text { Geometric- } \\
\text { Arithmetic }\end{array}$ & $\frac{2 \sqrt{i j}}{i+j}$ & $G A(G)$ & Inverse sum & $\frac{i j}{i+j}$ & $I S(G)$ \\
\hline $\begin{array}{l}\text { Sum- } \\
\text { Connectivity }\end{array}$ & $\frac{1}{\sqrt{i+j}}$ & $S C I(G)$ & Albertson & $|i-j|$ & $A l b(G)$ \\
\hline Harmonic & $\frac{2}{i+j}$ & $H I(G)$ & $\begin{array}{l}\text { Atom-Bond } \\
\text { connectivity }\end{array}$ & $\sqrt{\frac{i+j-2}{i j}}$ & $A B C G)$ \\
\hline First Zagreb & $i+j$ & $M_{1}(G)$ & $\begin{array}{l}\text { Symmetric } \\
\text { Division } \\
\text { Index }\end{array}$ & $\frac{i^{2}+j^{2}}{i j}$ & $S D(G)$ \\
\hline $\begin{array}{l}\text { Second } \\
\text { Zagreb }\end{array}$ & $i j$ & $M_{2}(G)$ & $\begin{array}{l}\text { Augmented } \\
\text { Zagreb }\end{array}$ & $\left(\frac{i j}{i+j-2}\right)^{3}$ & $A Z I(G)$ \\
\hline
\end{tabular}

Table 1: Some Important Formulae of degree based Topological indices

Theorem 1. Let ' $n$ ' be the number of rows in the Zig-Zag chain of 8-cycles of graph $Z_{8}(n)$, then Randicindex is given by $\chi\left(Z_{8}(n)\right)=\frac{13 n+2 \sqrt{6} n+3}{3}$

Proof: Randic Index is denoted by

$$
\chi\left(Z_{8}(n)\right) \frac{1}{\sqrt{i j}}
$$


Degree based topological indices of zig zag chain

$$
\begin{aligned}
= & \frac{1}{\sqrt{i j}}\{6 n+4\}+\frac{1}{\sqrt{i j}}\{4 n\}+\frac{1}{\sqrt{i j}}\{4 n-3\} \\
= & \frac{1}{\sqrt{2 * 2}}\{6 n+4\}+\frac{1}{\sqrt{2 * 3}}\{4 n\}+\frac{1}{\sqrt{3 * 3}}\{4 n-3\} \\
= & \frac{1}{\sqrt{4}}\{6 n+4\}+\frac{1}{\sqrt{6}}\{4 n\}+\frac{1}{\sqrt{3 * 3}}\{4 n-3\}=\frac{1}{\sqrt{4}}\{6 n+4\}+\frac{1}{\sqrt{6}}\{4 n\}+\frac{1}{\sqrt{9}}\{4 n-3\} \\
= & \frac{6 n+4}{2}+\frac{4 n}{\sqrt{6}}+\frac{4 n-3}{3}=\frac{3(6 n+4)+2(4 n-3)}{6}+\frac{4 n}{\sqrt{6}}=\frac{18 n+12+8 n-6}{6}+\frac{4 n}{\sqrt{6}} \\
= & \frac{26 n+6}{6}+\frac{4 n}{\sqrt{6}}=\frac{26 n+6}{\sqrt{6} \sqrt{6}}+\frac{4 n}{\sqrt{6}}=\frac{26 n+6+4 n(\sqrt{6})}{6}=\frac{13 n+2 \sqrt{6} n+3}{3} \\
& \chi\left(Z_{8}(n)\right)=\frac{13 n+2 \sqrt{6} n+3}{3}
\end{aligned}
$$

Theorem 2. Let ' $n$ ' be the number of rows in the Zig-Zag chain of 8-cycles of graph $\mathrm{Z}_{8}(\mathrm{n})$, then Geometric-Arithmetic index is given by $G A\left(Z_{8}(n)\right)=\frac{1}{5}(50 n+5+8 n \sqrt{6})$

Proof: Geometric - Arithmetic Index is denoted by

$$
\begin{aligned}
& G A\left(Z_{8}(n)\right)=\frac{2 \sqrt{i j}}{i+j} \\
& =\frac{2 \sqrt{i j}}{i+j}\{6 n+4\}+\frac{2 \sqrt{i j}}{i+j}\{4 n\}+\frac{2 \sqrt{i j}}{i+j}\{4 n-3\} \\
& =\frac{2 \sqrt{2 * 2}}{2+2}(6 n+4)+\frac{2 \sqrt{2 * 3}}{2+3}\{4 n\}+\frac{2 \sqrt{3 * 3}}{3+3}\{4 n-3\} \\
& =\frac{2 \sqrt{4}}{4}(6 n+4)+\frac{2 \sqrt{6}}{5}\{4 n\}+\frac{2 \sqrt{9}}{6}\{4 n-3\}=(6 n+4)+\frac{2 \sqrt{6}}{5}\{4 n\}+\{4 n-3\} \\
& =(6 n+4)+\frac{8 n \sqrt{6}}{5}+\{4 n-3\}=10 n+1+\frac{8 n \sqrt{6}}{5} \\
& G A\left(Z_{8}(n)\right)=\frac{1}{5}(50 n+5+8 n \sqrt{6})
\end{aligned}
$$

Theorem 3. Let ' $n$ ' be the number of rows in the Zig-Zag chain of 8-cycles of graph $\mathrm{Z}_{8}(\mathrm{n})$, then Sum-Connectivity index is given by

$$
\operatorname{SCI}\left(Z_{8}(n)\right)=\frac{3 \sqrt{30} n+2 \sqrt{30}-3 \sqrt{5}+4 \sqrt{6} n+4 \sqrt{5} n}{\sqrt{30}}
$$




\section{P.Gayathri ${ }^{1}$ and U.Priyanka ${ }^{2}$}

\section{Proof:}

Sum - Connectivity is denoted by $\operatorname{SCI}\left(Z_{8}(n)\right)=\frac{1}{\sqrt{i+j}}$

$$
\begin{aligned}
& =\frac{1}{\sqrt{i+j}}\{6 n+4\}+\frac{1}{\sqrt{i+j}}\{4 n\}+\frac{1}{\sqrt{i+j}}\{4 n-3\} \\
& =\frac{1}{\sqrt{2+2}}\{6 n+4\}+\frac{1}{\sqrt{2+3}}\{4 n\}+\frac{1}{\sqrt{3+3}}\{4 n-3\} \\
& =\frac{1}{\sqrt{4}}\{6 n+4\}+\frac{1}{\sqrt{5}}\{4 n\}+\frac{1}{\sqrt{3+3}}\{4 n-3\}=\frac{1}{2}\{6 n+4\}+\frac{1}{\sqrt{5}}\{4 n\}+\frac{1}{\sqrt{6}}\{4 n-3\} \\
& =\frac{6 n}{2}+\frac{4}{2}+\frac{4 n}{\sqrt{5}}+\frac{4 n}{\sqrt{6}}-\frac{3}{\sqrt{6}}=3 n+2-\frac{3}{\sqrt{6}}+\frac{4 n}{\sqrt{5}}+\frac{4 n}{\sqrt{6}}=3 n+2-\frac{3}{\sqrt{6}}+\frac{4 n}{\sqrt{5}}+\frac{4 n}{\sqrt{6}} \\
& \operatorname{SCI}\left(Z_{8}(n)\right)=\frac{3 \sqrt{30} n+2 \sqrt{30}-3 \sqrt{5}+4 \sqrt{6} n+4 \sqrt{5} n}{\sqrt{30}}
\end{aligned}
$$

Theorem 4. Let ' $n$ ' be the number of rows in the Zig-Zag chain of 8-cycles of graph $Z_{8}(n)$, then Harmonic index is given by $H I\left(Z_{8}(n)\right)=\frac{89 n+15}{15}$

\section{Proof:}

Harmonic index is denoted by $H I\left(Z_{8}(n)\right)=\frac{2}{i+j}$

$$
\begin{aligned}
& =\frac{2}{i+j}\{6 n+4\}+\frac{2}{i+j}\{4 n\}+\frac{2}{i+j}\{4 n-3\} \\
& =\frac{2}{2+2}\{6 n+4\}+\frac{2}{2+3}\{4 n\}+\frac{2}{3+3}\{4 n-3\} \\
& =\frac{2}{4}\{6 n+4\}+\frac{2}{5}\{4 n\}+\frac{2}{3+3}\{4 n-3\}=\frac{1}{2}\{6 n+4\}+\frac{2}{5}\{4 n\}+\frac{2}{3+3}\{4 n-3\} \\
& =\frac{6 n}{2}+\frac{4}{2}+\frac{8 n}{5}+\frac{8 n}{6}-\frac{6}{6}=3 n+2+\frac{8 n}{5}+\frac{4 n}{3}-1=3 n+1+\frac{8 n}{5}+\frac{4 n}{3} \\
& =\frac{45 n+15+20 n+24 n}{15} \\
& H I\left(Z_{8}(n)\right)=\frac{89 n+15}{15}
\end{aligned}
$$

Theorem 5. Let ' $n$ ' be the number of rows in the Zig-Zag chain of 8-cycles of graph $Z_{8}(n)$, then first Zagreb index is given by $M_{1}\left(Z_{8}(n)\right)=68 n-2$ 
Degree based topological indices of zig zag chain

Proof: First Zagreb Index is denoted by $M_{1}\left(Z_{8}(n)\right)=i+j$

$$
\begin{aligned}
& =(i+j)\{6 n+4\}+(i+j)\{4 n\}+(i+j)\{4 n-3\} \\
& =(2+2)\{6 n+4\}+(2+3)\{4 n\}+(3+3)\{4 n-3\} \\
& =(4)\{6 n+4\}+(5)\{4 n\}+(3+3)\{4 n-3\}=(4)\{6 n+4\}+\{20 n\}+(6)\{4 n-3\} \\
& =24 n+16+20 n+24 n-18 \\
& M_{1}\left(Z_{8}(n)\right)=68 n-2
\end{aligned}
$$

Theorem 6. Let ' $n$ ' be the number of rows in the Zig -Zag chain of 8-cycles of graph $\mathrm{Z}_{8}(\mathrm{n})$, then Second Zagreb index is given by $M_{2}\left(Z_{8}(n)\right)=84 n-11$

Proof: Second Zagreb index is denoted by $M_{2}\left(Z_{8}(n)\right)=i j$

$$
\begin{aligned}
& =(i j)\{6 n+4\}+(i j)\{4 n\}+(i j)\{4 n-3\}=(2 * 2)\{6 n+4\}+(2 * 3)\{4 n\}+(3 * 3)\{4 n-3\} \\
& =(4)\{6 n+4\}+(6)\{4 n\}+(3 * 3)\{4 n-3\}=24 n+16+24 n+36 n-27 \\
& M_{2}\left(Z_{8}(n)\right)=84 n-11
\end{aligned}
$$

Theorem 7. Let ' $n$ ' be the number of rows in the Zig -Zag chain of 8-cycles of graph $Z_{8}(n)$, then Second Modified Zagreb index is given by $M_{3}\left(Z_{8}(n)\right)=\frac{141 n}{54}-\frac{4}{3}$

Proof: Second Modified Zagreb index is denoted by $M_{3}\left(Z_{8}(n)\right)=\frac{1}{i j}$

$$
\begin{aligned}
& =\frac{1}{i j}\{6 n+4\}+\frac{1}{i j}\{4 n\}+\frac{1}{i j}\{4 n-3\}=\frac{1}{2 * 2}\{6 n+4\}+\frac{1}{2 * 3}\{4 n\}+\frac{1}{3 * 3}\{4 n-3\} \\
& =\frac{1}{4}\{6 n+4\}+\frac{1}{6}\{4 n\}+\frac{1}{3 * 3}\{4 n-3\}=\frac{6 n+4}{4}+\frac{4 n}{6}+\frac{4 n-3}{9}=\frac{3 n}{2}+1+\frac{2 n}{3}+\frac{4 n}{9}-\frac{3}{9} \\
& =\frac{3 n}{2}+\frac{2 n}{3}+\frac{4 n}{9}-\frac{1}{3}+1=\frac{3 n}{2}+\frac{2 n}{3}+\frac{4 n}{9}-\frac{4}{3}=\frac{9 n+4 n}{6}+\frac{4 n}{9}-\frac{4}{3}=\frac{13 n}{6}+\frac{4 n}{9}-\frac{4}{3} \\
& =\frac{117 n+24 n}{54}-\frac{4}{3} \\
& M_{3}\left(Z_{8}(n)\right)=\frac{141 n}{54}-\frac{4}{3}
\end{aligned}
$$

Theorem 8. Let ' $n$ ' be the number of rows in the Zig -Zag chain of 8-cycles of graph $\mathrm{Z}_{8}(\mathrm{n})$, then Inverse sum index is given by $I S\left(Z_{8}(n)\right)=\frac{168 n-5}{10}$

Proof: Inverse Sum Index is denoted by

$$
I S\left(Z_{8}(n)\right)=\frac{i j}{i+j}
$$




\section{P.Gayathri ${ }^{1}$ and U.Priyanka ${ }^{2}$}

$$
\begin{aligned}
& =\frac{i j}{i+j}\left\{(6 n+4\}+\frac{i j}{i+j}\{4 n\}+\frac{i j}{i+j}\{4 n-3\}\right. \\
& =\frac{2 * 2}{2+2}\{6 n+4\}+\frac{2 * 3}{2+3}\{4 n\}+\frac{3 * 3}{3+3}\{4 n-3\} \\
& =\frac{4}{4}\{6 n+4\}+\frac{6}{5}\{4 n\}+\frac{9}{6}\{4 n-3\}=6 n+4+\frac{24 n}{5}+\frac{36 n}{6}-\frac{27}{6} \\
& =6 n+4+\frac{24 n}{5}+6 n-\frac{9}{2}=6 n+6 n+\frac{24 n}{5}+4-\frac{9}{2}=12 n+\frac{24 n}{5}+4-\frac{9}{2}=\frac{84 n}{5}-\frac{1}{2} \\
& I S\left(Z_{8}(n)\right)=\frac{168 n-5}{10}
\end{aligned}
$$

Theorem 9. Let ' $n$ ' be the number of rows in the Zig -Zag chain of 8-cycles of graph $Z_{8}(\mathrm{n})$, then Albertson index is given by $A l b\left(Z_{8}(n)\right)=4 n$

Proof: Albertson index $A l b\left(Z_{8}(n)\right)=|i-j|$

$$
\begin{aligned}
& =|i-j|\{6 n+4\}+|i-j|\{4 n\}+|i-j|\{4 n-3\} \\
& =|2-2|\{6 n+4\}+|2-3|\{4 n\}+|3-3|\{4 n-3\}, \operatorname{Alb}\left(Z_{8}(n)\right)=4 n
\end{aligned}
$$

Theorem 10. Let ' $n$ ' be the number of rows in the Zig -Zag chain of 8-cycles of graph $\mathrm{Z}_{8}(\mathrm{n})$, then Atom-Bond connectivity index is given by

$$
A B C\left(Z_{8}(n)\right)=\frac{15 \sqrt{2} n+6 \sqrt{2}+8 n-6}{3}
$$

Proof: Atom Bomb Connectivity

$$
\begin{aligned}
& A B C\left(Z_{8}(n)\right)=\sqrt{\frac{i+j-2}{i j}} \\
& =\sqrt{\frac{i+j-2}{i j}}\{6 n+4\}+\sqrt{\frac{i+j-2}{i j}}\{4 n\}+\sqrt{\frac{i+j-2}{i j}}\{4 n-3\} \\
& =\sqrt{\frac{2+2-2}{2 * 2}}\{6 n+4\}+\sqrt{\frac{2+3-2}{2 * 3}}\{4 n\}+\sqrt{\frac{3+3-2}{3 * 3}}\{4 n-3\} \\
& =\sqrt{\frac{2}{4}}\{6 n+4\}+\sqrt{\frac{3}{6}}\{4 n\}+\sqrt{\frac{4}{9}}\{4 n-3\}=\sqrt{\frac{1}{2}}\{6 n+4\}+\sqrt{\frac{1}{2}}\{4 n\}+\frac{2}{3}\{4 n-3\} \\
& =\sqrt{\frac{1}{2}}\{6 n+4\}+\sqrt{\frac{1}{2}}\{4 n\}+\frac{2}{3}\{4 n-3\}=\frac{1}{\sqrt{2}}\{6 n+4\}+\frac{1}{\sqrt{2}}\{4 n\}+\frac{2}{3}\{4 n-3\} \\
& =\frac{6 n+4}{\sqrt{2}}+\frac{4 n}{\sqrt{2}}+\frac{2(4 n-3)}{3}=\frac{10 n+4}{\sqrt{2}}+\frac{8 n-6}{3}=\frac{2(5 n+2)}{\sqrt{2}}+\frac{8 n-6}{3} \\
& =\frac{\sqrt{2} \sqrt{2}(5 n+2)}{\sqrt{2}}+\frac{8 n-6}{3}=\sqrt{2}(5 n+2)+\frac{8 n-6}{3}
\end{aligned}
$$


Degree based topological indices of zig zag chain

$$
A B C\left(Z_{8}(n)\right)=\frac{15 \sqrt{2} n+6 \sqrt{2}+8 n-6}{3}
$$

Theorem 11. Let ' $n$ ' be the number of rows in the Zig -Zag chain of 8-cycles of graph $Z_{8}(n)$, then Symmetric Division Index is given by $S D\left(Z_{8}(n)\right)=\frac{86 n+6}{3}$

Proof: Symmetric Division Index is denoted by

$$
\begin{aligned}
& S D\left(Z_{8}(n)\right)=\frac{i^{2}+j^{2}}{i j} \\
& =\frac{i^{2}+j^{2}}{i j}\{6 n+4\}+\frac{i^{2}+j^{2}}{i j}\{4 n\}+\frac{i^{2}+j^{2}}{i j}\{4 n-3\} \\
& =\frac{(2)^{2}+(2)^{2}}{2 * 2}\{6 n+4\}+\frac{(2)^{2}+(3)^{2}}{2 * 3}\{4 n\}+\frac{(3)^{2}+(3)^{2}}{3 * 3}\{4 n-3\} \\
& =\frac{4+4}{4}\{6 n+4\}+\frac{4+9}{6}\{4 n\}+\frac{9+9}{9}\{4 n-3\}=\frac{8}{4}\{6 n+4\}+\frac{52 n}{6}+\frac{18}{9}\{4 n-3\} \\
& =12 n+8+8 n-6+\frac{52 n}{6}=20 n+2+\frac{52 n}{6}=\frac{120 n+12+52 n}{6}=\frac{60 n+2+26 n}{3} \\
& S D\left(Z_{8}(n)\right)=\frac{86 n+6}{3}
\end{aligned}
$$

Theorem 12. Let ' $n$ ' be the number of rows in the Zig -Zag chain of 8-cycles of graph $Z_{8}(n)$, then Augmented Zagreb index is given by $A Z I\left(Z_{8}(n)\right)=\frac{8036 n-139}{64}$

Proof: Augmented Zagreb index is denoted by

$$
\begin{aligned}
& A Z I\left(Z_{8}(n)\right)=\left(\frac{i j}{i+j-2}\right)^{3} \\
& =\left(\frac{i j}{i+j-2}\right)^{3}\{6 n+4\}+\left(\frac{i j}{i+j-2}\right)^{3}\{4 n\}+\left(\frac{i j}{i+j-2}\right)^{3}\{4 n-3\} \\
& =\left(\frac{2 * 2}{2+2-2}\right)^{3}\{6 n+4\}+\left(\frac{2 * 3}{2+3-2}\right)^{3}\{4 n\}+\left(\frac{3 * 3}{3+3-2}\right)^{3}\{4 n-3\} \\
& =\left(\frac{4}{2}\right)^{3}\{6 n+4\}+\left(\frac{6}{3}\right)^{3}\{4 n\}+\left(\frac{3 * 3}{4}\right)^{3}\{4 n-3\} \\
& =(2)^{3}\{6 n+4\}+(2)^{3}\{4 n\}+\left(\frac{9}{4}\right)^{3}\{4 n-3\} \\
& =(8)\{6 n+4\}+(8)\{4 n\}+\left(\frac{9}{4}\right)^{3}\{4 n-3\}=48 n+32+32 n+\left(\frac{729}{64}\right)^{3}\{4 n-3\}
\end{aligned}
$$




$$
\begin{gathered}
\text { P.Gayathri }{ }^{l} \text { and } \text { U.Priyanka }^{2} \\
=80 n+32+\left(\frac{729}{64}\right)^{3}\{4 n-3\}=\frac{5120 n+2048-2916 n-2187}{64} \\
\operatorname{AZI}\left(Z_{8}(n)\right)=\frac{8036 n-139}{64}
\end{gathered}
$$

\section{Conclusion}

Due to the quarrelsome evolving infections or viruses, a great deal of new diseases can be exposed every year. This necessitates us to develop more new drugs to indulgence new diseases. Topological indices were bring together to measure the medicinal properties of new medications which is principally popular in emerging areas. In our article, in terms of vertex degrees of molecular graph of drugs, we determined the well popular topological indices of certain molecular graphs which are widely appear in drug structures as an application for pharmacy engineering.

\section{REFERENCES}

1. A.Loghman and L. Badakhshiana, PI Polynomial of Zig - Zagpolyhex Nanotubes, Digest journal of Nanomaterials and Biostructure, 3(4) (2008) 299-302.

2. M.Baca, J.Horvathova, M.Mokrisova, A.Semanicova-Fenovckova and A.Suhanyiova, On topological indices of carbon nanotube network, Canadian Journal of Chemistry, 93(10) (2015) 1157-1160.

3. M.R.Farahani, A new version of Zagreb index of circumcoronene series of benzenoid, Chemical Physics Research Journal, 6(1) (2013) 27-33.

4. M.R.Farahani and M.P.Vlad, Computing first and second Zagreb index, first and second Zagreb polynomial of Capra-designed planar benzenoid series Can (C6), StudiaUbbChemia, 58(2) (2013) 133-142.

5. B.Furtula and I.Gutman, A forgotten topological index, Journal of Mathematical Chemistry, 53(4) (2015) 1184-1190.

6. W.Gao and M.R.Farahani, Computing the reverse eccentric connectivity index for certain family of nanocone and fullerene structures, Journal of Nanotechnology, 37(1) (2016) 1-6.

7. W.Gao and M.R.Farahani, Degree-based indices computation for special chemical molecular structures using edge dividing method, Applied Mathematics and Nonlinear Science, 1(1) (2015) 94-117.

8. W.Gao and L.Shi, Szeged related indices of unilateral polyomino chain and unilateral hexagonal chain, IAENG International Journal of Applied Mathematics, 45(2) (2015) $138-150$.

9. W.Gao and W.F.Wang, The fifth geometric-arithmetic index of bridge graph and carbon nanocones, Journal of Difference applications, 23 (2016) 100-109.

10. W.F.Xi and W.Gao, Geometric- Arithmetic Index and Zagreb Indices of certain special chemical molecular structures, Journal of chemistry, 26(11) (2014) 33973400 .

11. L.Yan, Y.Li, W.Gao and J.Li, PI Index for Some Special Graphs, Journal of Chemical and Pharmaceutical Research, 5(11) (2013) 260-265.

12. W.Gao, M.R.Farahani, Forgotten topological index of some drug structures, Journal of Saudi Pharmaceutical, 24(3) (2016) 258-264. 
Degree based topological indices of zig zag chain

13. M.H.Khalifeth, H.Yousefi -Azari and A.R.Ashrafi, Vertex and Edge PI Indices of Cartesian Product graph, Discrete Applied Mathematics, 156(10) (2008) 1780-1789.

14. P.Gayathri, U.Priyanka, S.Sandhiya, S.Sunandha and K.R.Subramanian, "MPolynomials of Penta-Chains, Journal of Ultra Scientist of Physical Sciences, 29(4) (2017) 164-168.

15. P.Gayathri, U.Priyanka and S.Sandhiya, A significant computation for finding PI index of Phenylene, Journal of Ultra Chemistry, 13(3) (2017) 60-64.

16. M.Ghorbani and M.Ghazi, Computing some topological indices of triangular benzenoid, Digest Journal of Nanomaterials Biostructure, 5(4) (2010) 837-841.

17. P.Gayathri and U.Priyanka, Degree Based Topological Indices of Banana Tree Graph, International Journal of Current Research and Modern Education, Special Issue, NCETM (2017) 13-24.

18. P.Gayathri and U.Priyanka, Degree based topological indices of Linear Phenylene, International Journal of Innovative Research in Science, Engineering and Technology, 6(8) (2017) 16986-16997 